Research Article

\title{
Decoupling Control of a Multiaxis Hydraulic Servo Shaking Table Based on Dynamic Model
}

\author{
Qitao Huang $\mathbb{D}$, Peng Wang $\mathbb{D}$, Yuhao Wang $\mathbb{D}$, and Qinjun Yang $\mathbb{C}$ \\ School of Mechatronics Engineering, Harbin Institute of Technology, Harbin 150001, China \\ Correspondence should be addressed to Qitao Huang; huangqitao@hit.edu.cn
}

Received 17 May 2021; Revised 1 October 2021; Accepted 27 October 2021; Published 23 November 2021

Academic Editor: N. A. Saeed

Copyright (c) 2021 Qitao Huang et al. This is an open access article distributed under the Creative Commons Attribution License, which permits unrestricted use, distribution, and reproduction in any medium, provided the original work is properly cited.

\begin{abstract}
Hydraulic servo shaking table is an essential testing facility to simulate the actual vibration situation in real time. As a parallel mechanism, multiaxis hydraulic servo shaking table shows strong coupling characteristic among different degrees of freedom. When the multiaxis hydraulic shaking table moves to one direction, some unnecessary related motions will appear in other directions, which seriously affect the control performance. An effective approach to decouple motions in command direction and in unnecessary related directions is an urgent need for a higher precision control performance. In this work, the coupling phenomena and reasons of the multiaxis hydraulic servo table are analyzed based on dynamic model of a multiaxis hydraulic servo shaking table. In this regard, multiaxis hydraulic servo shaking table with strong coupling within the physical space is transformed into a set of single-input single-output systems that are independent of each other in the modal space. A decoupling control strategy is proposed in modal space to restrain the coupling motions. Simulation and experimental results show that the proposed control strategy can effectively improve the control performance and the decoupling effect.
\end{abstract}

\section{Introduction}

Hydraulic servo shaking table is widely used to artificially simulate the desired motion exerted on the test specimen owing to its high power to weight ratio, large load carrying capability, fast response, and high stiffness [1-4]. Therefore, it is extensively employed in seismic engineering [5], automobile industry [6], and structure fatigue testing [7]. For the multiaxis hydraulic servo shaking table, the number of hydraulic cylinders is greater than the number of degrees of freedom, and the complex dynamic characteristics make strong coupling effect between the different degrees of freedom, which results in deviation from the desired motion [8-11]. In addition, the nonlinearity of multiaxis hydraulic servo shaking table and the produced coupling force during the force loading process deteriorate the control performance. Hence, it is essential to design a new controller that alleviates the coupling effect and improve the control performance.

The motion of each hydraulic cylinder of the parallel mechanism generates coupling, so the joint motion of each branch is accompanied by other hydraulic cylinders; otherwise, the immobile hydraulic cylinder will limit the motion of the multiaxis hydraulic servo shaking table [12]. Tagawa and Kajiwara studied the three-variable control technique to improve the stability and tracking performance of six-axis hydraulic servo shaking table [13]. Guan and Plummer proposed accelerating decoupling control strategy based on modal control and inverse dynamics to solve the inner force coupling problem of the 6-degree-of-freedom electrohydraulic shaking table [14]. Plummer presented model-based motion control method to decouple each control axis of multiaxis servo hydraulic shaking table, where linear parameter varying modal control approach is used to control exclusively the modes of hydraulic actuator with the modal decomposition repeated at each time step to account for parameter variation [15]. Shen et al. designed an integrated controller including pressure stabilizing controller and proportional-integral-derivative controller with the transformation matrix to decouple the internal coupling of the sixdegree-of-freedom electrohydraulic shaking table [16]. Yang et al. proposed a decoupled-space control framework for multidegree freedom parallel robotic, where singular value 
decomposition algorithm is used to realize the decoupling of the control apace [17]. Zhao et al. proposed modal space control method to eliminate the strong coupling problem of degree of freedom space channels, where the coupling physical space is transformed into the decoupling modal space by using the weighted orthogonality of the main mode with respect to mass matrix and stiffness matrix [18].

As an important method, the conventional proportionalintegral-derivative controller cannot cover the control requirements of the increasingly complex hydraulic servo system [19]. Thus, a variety of modern control methods have been developed to improve the control performance of multiaxis, especially 6-DOF hydraulic servo shaking table [20]. Shen et al. proposed inverse transfer function of the system based on three-variable controller and internal model control to improve the control accuracy of electrohydraulic system [21]. Zhang et al. utilized servo control and offline iterative control based on dynamic model to realize decoupling control of multiaxis seismic testing table [22]. Tian et al. proposed modal space controller based on inertial parameter identification method to alleviate the influence of coupling and improve the high-performance motion control of six-degree-of-freedom parallel manipulator [23]. Guan analyzed the kinematics and the pose control structure of six-degree-of-freedom vibration table, and the method using forward solution and Jacobian matrix rather than the degree of freedom composition and decomposition matrixes improving the independent control performance of each actuator in the low frequency band [24].

As mentioned above, a higher accuracy representation of input command is the main function to shaking table. This work includes the following creative contributions:

(1) The coupling of the multiaxis hydraulic servo table in different directions is analyzed based on dynamic model

(2) The sources of coupling motion are traced, and corresponding basic suggestions are provided

(3) The dynamic model is transformed into modal space, where the coupling system is transformed to a set of single-input single-output systems

(4) A decoupling control strategy is proposed to avoid the coupling, which is verified in simulation and experiments

The remainder of this paper is organized as follows. The experimental set of multiaxis hydraulic servo shaking table is introduced in Section "Experimental Setup." Section "Mathematical Modelling" presents the dynamic modelling and coupling characteristic. The controller design is designed in Section "Controller Design." The simulation and experiment are performed to verify the performance of the designed controller in Section "Simulation and Experimental Results." Finally, main conclusions are presented.

\section{Experimental Setup}

The hydraulic servo shaking table mainly consists of motion table, hydraulic actuator, and servo control system as shown in Figure 1. Hydraulic actuator adopted Haechen Inc. with $220 \mathrm{~mm}$ working stroke, flow-controlled servo-valve manufactured by Moog Inc. D79-200. The motion table is controlled by six-degree-of-freedom using eight doubleended hydraulic actuators. In order to eliminate the shortterm fluctuation of oil supply pressure in the hydraulic pipeline, the system is also equipped with 8 groups of accumulators with the highest working pressure of $31.5 \mathrm{MPa}$. In addition, linear variable differential transformer, accelerometer, and pressure transducer provide the real-time control signal of the control system. The main parameters of multiaxis hydraulic servo shaking table are listed in Table 1.

The aim of the shaking table is to reproduce the input acceleration signal at the control point of the motion platform. The data from each sensor is transmitted to the control signal of computer through the sensor system [25]. The schematic diagram of the multiaxis hydraulic servo shaking table is shown is Figure 2, where the program is compiled in the Simulink environment of the host computer. The human-computer interface produced by LabVIEW software can easily adjust the parameters of the control program and display the running state the multiaxis hydraulic servo shaking table. The host computer transfers the program to the target computer and compiles it into an executable C language file through XPC-Target system [26]. The target computer converts the control signal to the hydraulic system for driving the shaking table through D/A converter. The signal modulator transmits the sensor signal of the shaking table to the target computer through A/D converter [27].

\section{Mathematical Modelling}

3.1. The Dynamic Model. The overall structure of multiaxis hydraulic servo shaking table is shown in Figure 3. In order to describe the movement of the motion table, two coordinate systems are established, where the static coordinate system $O-X Y Z$ is fixed on the bottom platform, the moving coordinate system $O^{\prime}-X^{\prime} Y^{\prime} Z^{\prime}$ is fixed on the motion table, and $O$ and $O^{\prime}$ are the center of the motion table and the barycenter of motion table. Eight hydraulic cylinders are represented by $x_{i}, y_{i}(i=1,2)$ and $z_{j}(j=1,2,3,4)$, respectively. The motion table is connected to the bottom and the reaction wall by the eight hydraulic cylinders.

For the hydraulic cylinder output force, the direction of output force and the direction of hydraulic cylinder are identical. The force of each hydraulic actuator can be expressed as

$$
f_{a}=\left[\begin{array}{llllll}
f_{a_{1}} & f_{a_{2}} & f_{a_{3}} & f_{a_{4}} & f_{a_{5}} & f_{a_{6}}
\end{array}\right] .
$$

Assuming that the force of hydraulic actuator applied to the shaking table is a constant, the load of motion table is given as follows:

$$
\vec{f}_{a}+m \vec{g}==m_{3 \times 3} \ddot{t},
$$

where $m_{3 \times 3}=\operatorname{diag}\left[\begin{array}{lll}m & m & m\end{array}\right]$ and $\vec{g}=\left[\begin{array}{lll}0 & 0 & -g\end{array}\right]^{T}$.

The moment of momentum of shaking table can be expressed as 


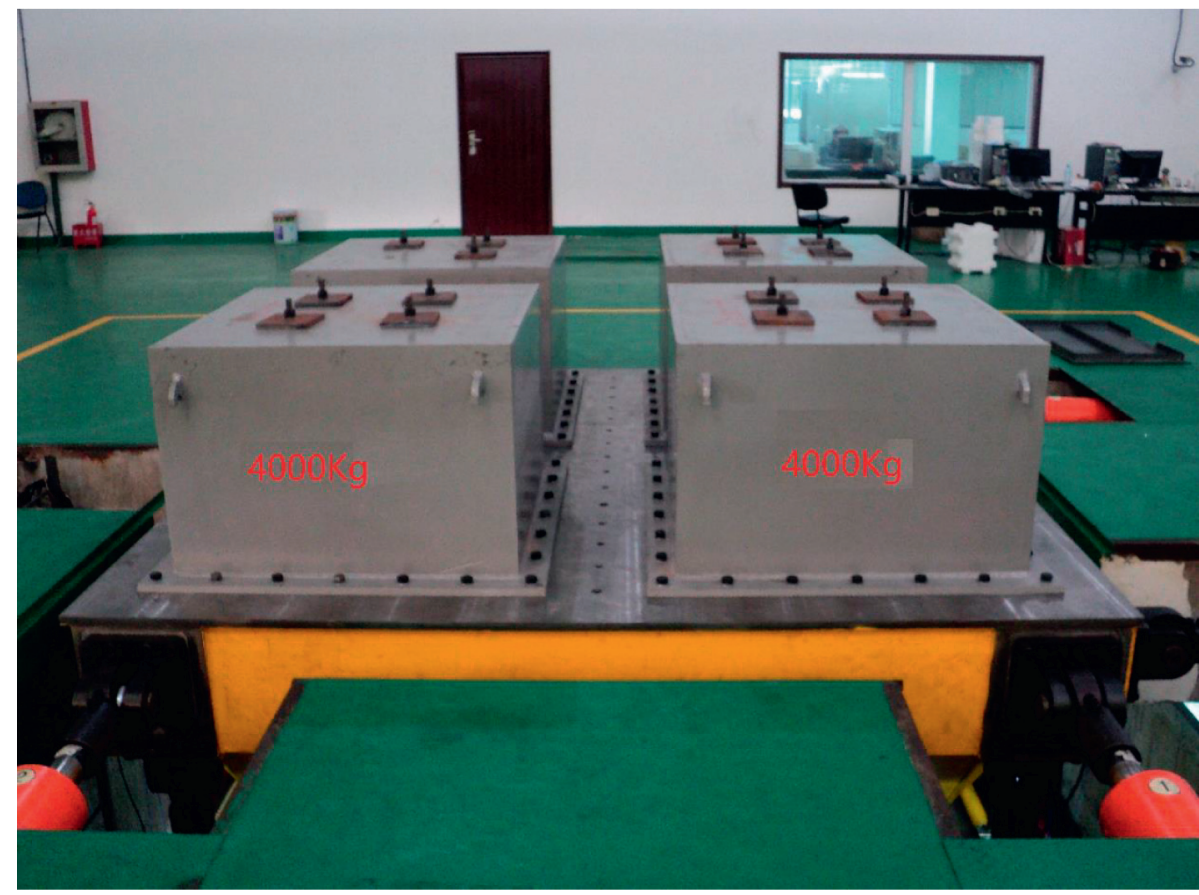

FIGURE 1: Multiaxis hydraulic servo shaking table experimental setup.

TABLE 1: Main parameters of $h$ multiaxis hydraulic servo shaking table.

\begin{tabular}{lc}
\hline Parameters & Value \\
\hline Platform size & $3.5 \mathrm{~m} \times 3.5 \mathrm{~m}$ \\
Motion table weight & $10000 \mathrm{~kg}$ \\
Degree of freedom & $X, Y, Z, R x, R y, R z$ \\
Maximum displacement & $X: \pm 220 \mathrm{~mm}, Y: \pm 220 \mathrm{~mm}, Z: \pm 220 \mathrm{~mm}$ \\
Maximum displacement & $X: \pm 3 \mathrm{~g}, Y: \pm 3 \mathrm{~g}, Z: \pm 10 \mathrm{~g}$ \\
\hline
\end{tabular}

$$
\vec{L}=\int \vec{r} \times \vec{v} \mathrm{~d} m=\int \vec{r} \times(\vec{\omega} \times \vec{r}) \mathrm{d} m
$$

where $\vec{\omega}$ is the instantaneous angular velocity vector, $\vec{r}$ is the radius vector, and $d m$ is the mass element.

The moment of inertia is defined as

$$
\left\{\begin{array}{l}
J_{x x}=\int\left(z^{2}+y^{2}\right) \mathrm{d} m, \quad J_{x y}=\int x y \mathrm{~d} m, \\
J_{y y}=\int\left(z^{2}+x^{2}\right) \mathrm{d} m, \quad J_{x z}=\int x z \mathrm{~d} m, \\
J_{z z}=\int\left(x^{2}+y^{2}\right) \mathrm{d} m,
\end{array}\right.
$$

Then, the inertia moment matrix can be written as

$$
J=\left[\begin{array}{ccc}
J_{x x} & -J_{x y} & -J_{x z} \\
-J_{y x} & J_{y y} & -J_{y z} \\
-J_{z x} & -J_{z y} & J_{z z}
\end{array}\right]
$$

According to the cross-product formula, (3) can be rewritten as

$$
\vec{L}=\int r^{2} \vec{E}-\vec{r} \vec{r} d m \cdot \vec{\omega}=J \cdot \vec{\omega}
$$

where $\vec{E}=\vec{i} \cdot \vec{i}+\vec{j} \cdot \vec{j}+\vec{k} \cdot \vec{k}$.

Although the inertia matrices of the different points are different, the relationship between them can be expressed as

$$
J_{O}^{0}=J_{C}^{0}+m \times\left[\begin{array}{ccc}
z_{C}^{2}+y_{C}^{2} & -x_{C} y_{C} & -x_{C} z_{C} \\
-x_{C} y_{C} & x_{C}^{2}+z_{C}^{2} & -y_{C} z_{C} \\
-x_{C} z_{C} & -y_{C} z_{C} & x_{C}^{2}+y_{C}^{2}
\end{array}\right],
$$

where $J_{O}^{0}$ is the matrix of rotational inertial centered $O, J_{C}^{0}$ is the matrix of rotational inertial centered $C$, and $x_{C}, y_{C}, z_{C}$ are the center of mass coordinate $C$ in the $O-X Y Z$ coordinate system.

For the same point, the inertia matrix is different in the different direction. The relationship of different coordinate system can be expressed as

$$
J_{O}^{0}=A_{01} J_{O}^{1} A_{10}
$$

According to Euler's equation, the moment of momentum equation can be expressed as

$$
\vec{M}_{O}=\dot{\vec{L}}_{O}=\dot{\vec{L}}_{O}+\vec{\omega} \times \vec{L}_{O}
$$

where $\stackrel{\circ}{L}_{O}=\left(J_{x x} \omega_{x} J_{y y} \omega_{y} J_{z z} \omega_{z}\right)^{T}$ is the inertia moment and $\vec{L}_{O}$ represents the relative derivative of the moment of momentum of a relatively fixed coordinate system. 


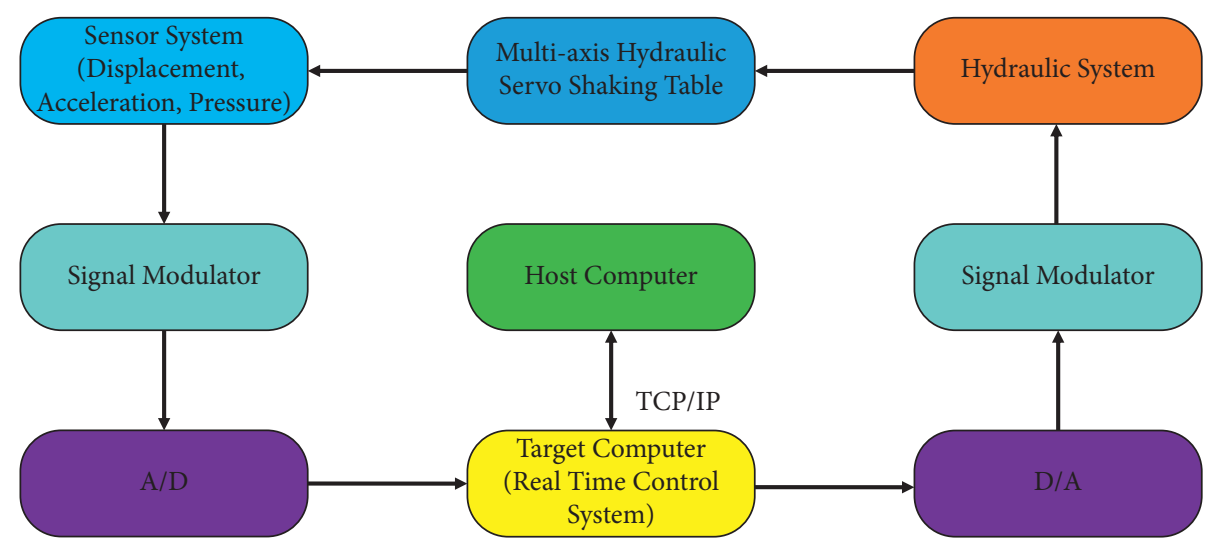

FIGURE 2: Schematic diagram of the multiaxis hydraulic servo shaking table.

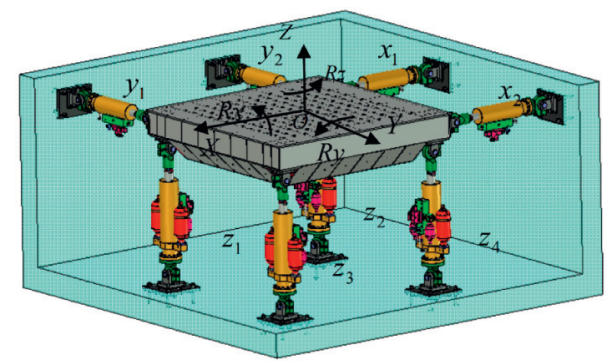

FIGURE 3: Overall structure of multiaxis hydraulic servo shaking table.

$$
\vec{M}_{O}=\dot{\vec{L}}_{O}=\dot{\vec{L}}_{O}+\vec{\omega} \times \vec{L}_{O}=\left(A_{01} A^{p} \times L_{n}\right) \cdot f_{a}=A_{01} J_{O}^{\prime} \dot{\omega}^{\prime}+A_{01} \omega^{\prime} \times A_{01} J_{O}^{\prime} \omega, \prime
$$

where $J_{O}^{\prime}$ represents the central principal inertia matrix through the center of mass of the relative fixed coordinate system, and $\omega \prime$ represents the projection of the angular velocity of the upper platform onto the fixed coordinate system.

The unified dynamics equation of single rigid body can be expressed as follows:

$$
\left[\begin{array}{c}
L_{n} \\
A_{01} A^{p} \times L_{n}
\end{array}\right]_{(6 \times 8)} \cdot f_{a(8 \times 1)}=\left[\begin{array}{cc}
m E_{(3 \times 3)} & 0 \\
0 & A_{01} J^{\prime}
\end{array}\right][\ddot{t} \dot{\omega} \prime]+\left[\begin{array}{cc}
0 & 0 \\
0 & A_{01} \omega \prime \times A_{01} J_{O}^{\prime}
\end{array}\right]\left[\begin{array}{c}
\dot{t} \\
\omega \prime
\end{array}\right]+\left[\begin{array}{c}
-m \vec{g} \\
0
\end{array}\right] .
$$

Because the motion between the upper platform and the lower is considered to be relatively small, it is considered to be approximately the unit matrix, and the approximate mass matrix is generally regarded as the constant value matrix. Similarly, the Coriolis force term is so small that it can be ignored. The influence of gravity in the dynamic equation can be offset by adjusting the force provided by the static pressure support. Equation (11) can be simplified as

$$
\left[\begin{array}{c}
L_{n} \\
A_{01} A^{p} \times L_{n}
\end{array}\right]_{(6 \times 8)} \cdot f_{a(8 \times 1)}=\left[\begin{array}{cc}
m E_{(3 \times 3)} & \\
& J_{O}^{\prime}
\end{array}\right]\left[\ddot{t} \dot{\omega}^{\prime}\right] .
$$

Therefore, it can be approximately considered that there is a one-to-one correspondence between the six generalized degrees of freedom directional forces and the generalized acceleration of the upper platform. It can be approximately considered as an uncoupled system when the center of mass coincides with the control point.

However, Euler equation cannot be simplified as the center of mass of the upper platform when the upper platform is loaded, and the control point is still the center of mass of the upper platform. According to the vector relationship, the relative acceleration of control point can be expressed as 


$$
\ddot{\vec{r}}_{D}=\ddot{\vec{r}}_{O}+\dot{\vec{\omega}} \times \vec{r}_{O D}+\vec{\omega} \times\left(\vec{\omega} \times \vec{r}_{O D}\right),
$$

where $D$ represents the center of mass of the upper platform and $C$ represents the center of mass of the load on the platform.

The kinetic equation with eccentric load can be written as

$$
M \ddot{\vec{r}}_{D}=M \ddot{\vec{r}}_{O}+M\left(\dot{\vec{\omega}} \times \vec{r}_{O D}\right)+M\left(\vec{\omega} \times\left(\vec{\omega} \times \vec{r}_{O D}\right)\right)
$$

where $m=m_{1}+m_{2}$, in which $m_{1}$ and $m_{2}$ are the mass of upper platform and lower platform, $M=\operatorname{diag}(m, m, m)$.

The force of the hydraulic actuator against the platform can be expressed as

$$
F=M \ddot{\vec{r}}_{D}^{0}-M\left(\left(A_{01} \dot{\vec{\omega}}^{1}\right) \times \vec{r}_{O D}^{0}\right)-M\left(\vec{\omega}^{0} \times A_{01} \vec{\omega}^{1}\right) \times \vec{r}_{O D}^{0}-M\left(\vec{\omega}^{0} \times\left(\vec{\omega}^{0} \times \vec{r}_{O D}^{0}\right)\right) .
$$

Ignoring the velocity term, equation (15) can be written as

$$
F=M\left[\ddot{x}_{D} \ddot{y}_{D} \ddot{z}_{D}\right]+M\left(A_{01}\left[\begin{array}{c}
\dot{\omega}_{x} \\
\dot{\omega}_{y} \\
\dot{\omega}_{z}
\end{array}\right] \times\left[\begin{array}{c}
x_{0} \\
y_{0} \\
z_{0}
\end{array}\right]\right) .
$$

Equation (16) can be rewritten as

$$
F=\left[\begin{array}{ccc}
m & 0 & 0 \\
0 & m & 0 \\
0 & 0 & m
\end{array},\left[\begin{array}{ccc}
0 & m z_{0} & -m y_{0} \\
-m z_{0} & 0 & m x_{0} \\
m y_{0} & -m x_{0} & 0
\end{array}\right] \cdot A_{01}\right] \ddot{q},
$$

where $\ddot{q}$ represents the generalized acceleration at the control point of the upper platform.

Therefore, it can be seen that the coupling force exerted by the system in one translational direction can be output in other directions when the upper platform has a mass.

$$
\begin{aligned}
\vec{M}_{D C} & =\dot{\vec{L}}_{C}^{\prime}+\dot{\vec{d}}_{D C} \times \vec{p}+\vec{d}_{D C} \times \dot{\vec{p}}+\vec{v}_{D} \times \vec{p} \\
& =\dot{\vec{L}}_{C}^{\prime}+\left(\dot{\vec{d}}_{D C}+\vec{v}_{D}\right) \times \vec{p}+\vec{d}_{D C} \times \dot{\vec{p}}=\dot{\vec{L}}_{C}^{\prime}+\vec{d}_{D C} \times \dot{\vec{p}} .
\end{aligned}
$$

The coupled force equation of multiaxis hydraulic servo shaking table can be deduced as

$$
F=\left[\begin{array}{cccccc}
m & 0 & 0 & 0 & m_{2} z_{C} & -m_{2} y_{C} \\
0 & m & 0 & -m_{2} z_{C} & 0 & m_{2} x_{C} \\
0 & 0 & m & m_{2} y_{C} & -m_{2} x_{C} & 0 \\
0 & -m_{2} z_{C} & m_{2} y_{C} & I_{x}+m_{2}\left(y_{C}^{2}+z_{C}^{2}\right) & -m_{2} x_{C} y_{C} & -m_{2} x_{C} z_{C} \\
m_{2} z_{C} & 0 & -m_{2} x_{C} & -m_{2} x_{C} y_{C} & I_{y}+m_{2}\left(x_{C}^{2}+z_{C}^{2}\right) & -m_{2} y_{C} z_{C} \\
-m_{2} y_{C} & m_{2} x_{C} & 0 & -m_{2} x_{C} z_{C} & -m_{2} y_{C} z_{C} & I_{z}+m_{2}\left(x_{C}^{2}+y_{C}^{2}\right)
\end{array}\right]\left[\ddot{x} \ddot{y} \ddot{z} \dot{\omega}_{x} \dot{\omega}_{y} \dot{\omega}_{z}\right] .
$$

3.2. The Analysis of Coupling Characteristic. The coordinate of the center of the load in the fixed coordinate system is $(0$, $0,0.8375$ ). The coupling output of $R x$ when $y$ is input direction is shown in Figure 4. Random acceleration signal in the direction of $Y$ degree of freedom with the peak value of $1.1 \mathrm{~m} / \mathrm{s}^{2}$ is input in the shaking table, and the coupling output can be seen in the direction of $R x$. In the absence of coupling, the output of this degree of freedom should be zero. The corresponding frequency domain waveform with $Y$ input and $R x$ output is shown in Figure 4(b). It can be seen that the coupling on the degree of freedom increases with the increase of frequency.

The center of load in the fixed coordinate is $(-0.86 \mathrm{~m}$, $-0.86 \mathrm{~m}, 0.8375 \mathrm{~m})$. In the $z$ direction, the input is a random acceleration signal with a peak value of $2.2 \mathrm{~m} / \mathrm{s}^{2}$, and the input of the rest degrees of freedom is equal to zero. In this case, the coupling output appears in the $R x$ direction. The coupling output of $R x$ when $z$ is input direction is shown in Figure 5. In the absence of coupling, the output of $R x$ direction should be zero. It can be seen from frequency domain that the coupling on the degree of $R x$ freedom increases with the increase of frequency.

The coupling output of $R x$ when $y$ is input direction is shown in Figure 6. In the direction of $Y$ degrees of freedom, input random acceleration signals with the peak value of $1.1 \mathrm{~m} / \mathrm{s}^{2}$, and the rest of the degrees of input signal are zero. At this time, the system has no load on the platform, and coupling output will be generated in the 


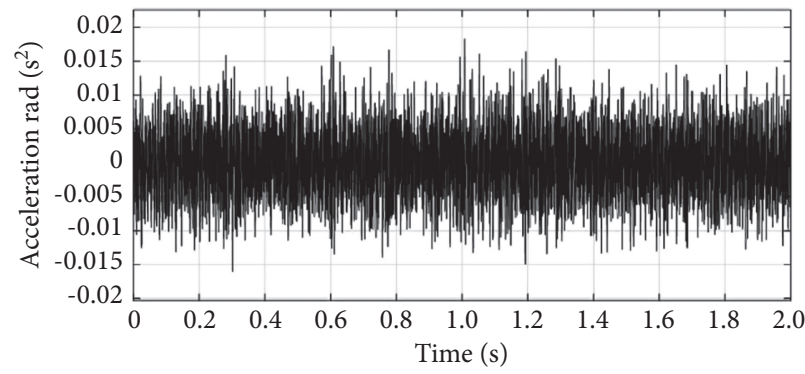

(a)

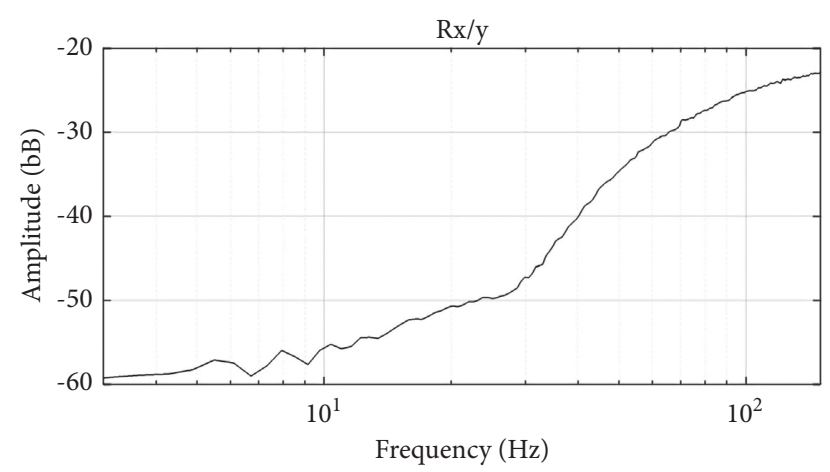

(b)

FIgURE 4: The coupling output of $R x$ when $y$ is input direction. (a) Acceleration and (b) frequency domain waveform.

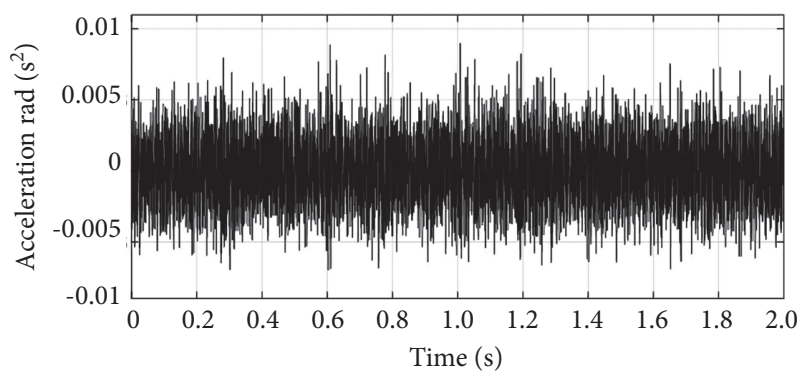

(a)

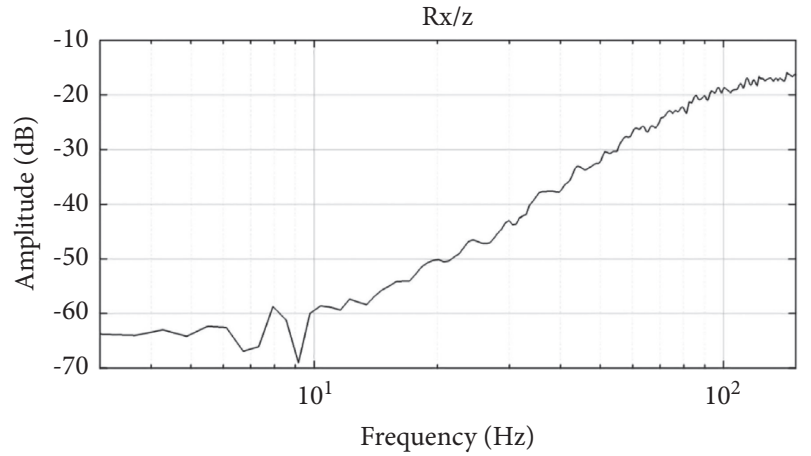

(b)

Figure 5: The coupling output of $R x$ when $z$ is input direction. (a) Acceleration and (b) frequency domain waveform.

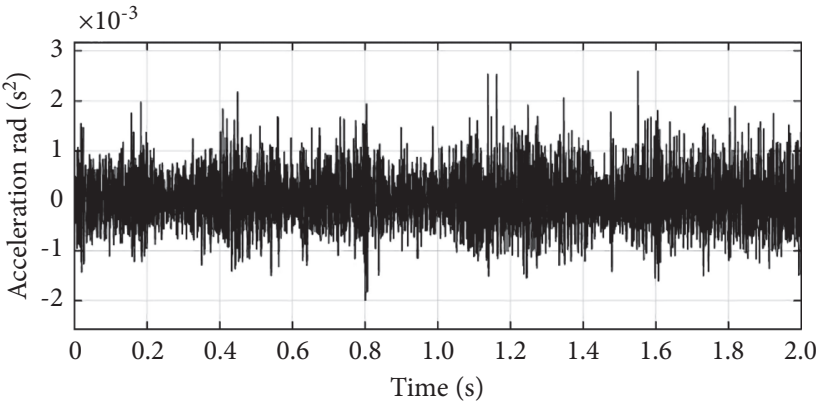

(a)

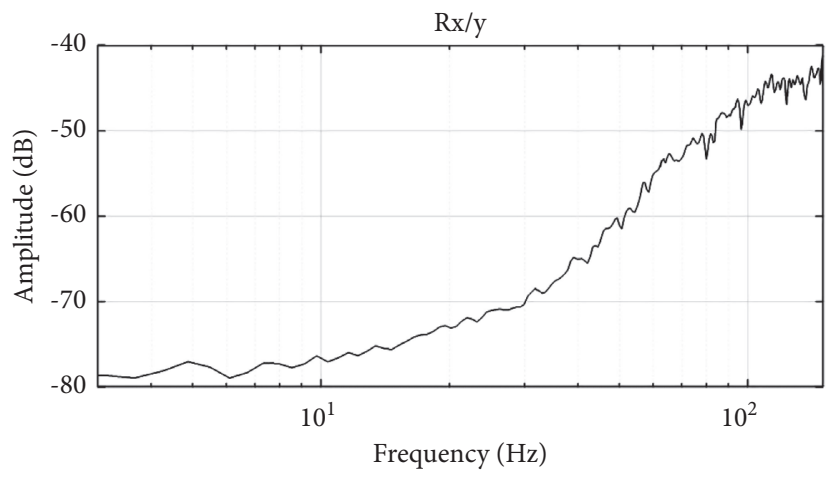

(b)

Figure 6: The coupling output of $R x$ when $y$ is input direction. (a) Acceleration and (b) frequency domain waveform.

direction of $R x$. In the case of no coupling, the output of $R x$ degree of freedom should be zero. It can be seen from the frequency domain that the coupling on the degree of freedom increases with the increase of frequency. But compared with the coupling caused by eccentricity of load, the coupling caused by inertia force of hydraulic cylinder is smaller.

\section{Controller Design}

4.1. The Analysis of Coupling Characteristic. The coupling output of the shaking table of each degree of freedom reduces the control precision and even destroys structural in serious cases. The decoupling control is needed to reduce the coupling output between the system degrees of freedom and 
increase the precision of waveform reproduction. The coupling output of each degree of freedom of the system mainly comes from the coupling caused by the geometric structure deviation; the coupling caused by the difference in the control performance of the hydraulic system of each leg; the coupling caused by internal force of redundant shaking table; the coupling caused by elastic foundation and elastic hinge; the coupling caused by eccentricity of platform load; the coupling caused by control point migration.

The coupling caused by the geometric structure deviation can be solved by improving the machining precision of mechanical parts and the level of assembly technology. The performance difference between actuators can be solved by selecting the high performance of servo-valves and servo cylinders. For the dynamics coupling, it is mainly caused by the off-diagonally dominant characteristic of mass matrix. For the dynamics coupling and the internal coupling, it is necessary to design the corresponding decoupling controller to reduce the coupling output of system.

For the multiaxis hydraulic servo shaking table, it has eight forces input, but only six generalized degrees of freedom output. Therefore, some forces will become internal forces of the system. If the internal forces are large enough, the system cannot move, and the mechanical parts of the system will be damaged.

The output force of hydraulic cylinder can be expressed as

$$
f_{a(8 \times 1)}=f_{a(8 \times 1)}^{*}+\Delta f_{a(8 \times 1)},
$$

where $f_{a(8 \times 1)}^{*}$ is the minimum output force of hydraulic cylinder and $\Delta f_{a(8 \times 1)}$ is the coupling output force of eight hydraulic cylinders.

The decoupling of internal forces can be realized by putting it into eight actuators as the control signal of internal force coupling. The decoupling equation can be expressed as

$$
\left\{\begin{array}{l}
J_{l q}^{T} \cdot f_{a(8 \times 1)}^{*}=F_{(6 \times 1)} \\
J_{l q}^{T} \cdot \Delta f_{a(8 \times 1)}=0
\end{array},\right.
$$

where $J_{l q}^{T}$ is Jacobian matrix.

As $J_{l q}^{T}$ is not a square matrix, the minimum output force of hydraulic cylinder can be deduced as

$$
f_{a(8 \times 1)}^{*}=\left(J_{l q}^{T}\right)^{-1} \cdot F=J_{l q}\left(J_{l q}^{T} J_{l q}\right)^{-1}\left(J_{l q}^{T}\right)^{-1} \cdot F .
$$

Then, the coupling output force of hydraulic cylinders can be expressed as

$$
\Delta f_{a(8 \times 1)}=f_{a(8 \times 1)}-\left(J_{l q}\left(J_{l q}^{T} J_{l q}\right)^{-1}\right) J_{l q}^{T} f_{a(8 \times 1)} .
$$

4.2. The Design of Decoupling Controller. The dynamic equation reflects the relationship between the output force and acceleration of each degree of freedom of the system. As the multiaxis hydraulic servo shaking table has a static equilibrium device, the influence of gravity on the dynamic equation can be ignored. As the amplitude of the shaking table is small, the Coriolis force term has less influence on the dynamic equation, so it can also be ignored. Because the mass matrix is off-diagonal dominant, there is no one-to-one correspondence between the acceleration of each degree of freedom and the generalized force of each degree of freedom, thus resulting in the coupling of the output of the system.

The mass matrix can be divided into two parts: the elements on the diagonal constitute an uncoupled mass matrix, and the elements off the diagonal constitute a coupled mass matrix. It is

$$
M=M_{1}+M^{*},
$$

where $M$ is the mass matrix of shaking table, $M_{1}$ is the uncoupled mass matrix of diagonal element, and $M^{*}$ is the coupled mass matrix of off-diagonal element.

The generalized force equation can be expressed as

$$
F=F_{1}+F^{*}=M_{1} q+M^{*} \ddot{q},
$$

where $F_{1}$ is coupling force, $F^{*}$ is uncoupling force, and $q$ is generalized displacement.

After the coupling force of the shaking table is obtained, the six-dimensional coupling force signal is converted into the coupling force signal on eight hydraulic cylinders. The coupling force can be obtained by

$$
f^{*}=\left[J_{l q}^{T}\right]^{-1} F^{*}
$$

The dynamic decoupling can be achieved when the coupling force signal is feedback to the input electrical signal of eight hydraulic cylinders.

\section{Simulation and Experimental Results}

5.1. Simulation Results. Figure 7 shows the comparison of random signal before and after decoupling without load when the input signal is a random acceleration signal with a peak value of $1.1 \mathrm{~m} / \mathrm{s}^{2}$ in the direction of $Y$ degrees of freedom. It can be seen from time domain that coupling signal output appeared in the direction of $R x$. However, the coupling output of the system decreases when the decoupling controller is added. As can be seen from the frequency domain, the output coupling between $y$ direction and $R x$ direction is small before system decoupling, when the upper platform has no load, and the decoupling controller can reduce the coupling by $10 \mathrm{~dB}$ on average.

In Figure 8, a shaking table is shown with eccentric load. Figure 9 shows the comparison of random signal before and after decoupling with eccentric load when the input signal is a random acceleration signal with a peak value of $1.1 \mathrm{~m} / \mathrm{s}^{2}$ in the direction of $Y$ degrees of freedom. It can be seen from time domain that coupling signal output appeared in the direction of $R x$. Time domain result shows that coupled output of the system decreases when the decoupling controller is applied. As can be seen from the frequency domain that the coupling output of $R x$ direction is greater than that without load when $z$ direction has eccentric load, and the decoupling controller can reduce coupling by $10 \mathrm{~dB}$ on average.

Figure 10 shows the comparison of random signal before and after decoupling with eccentric load when the input 


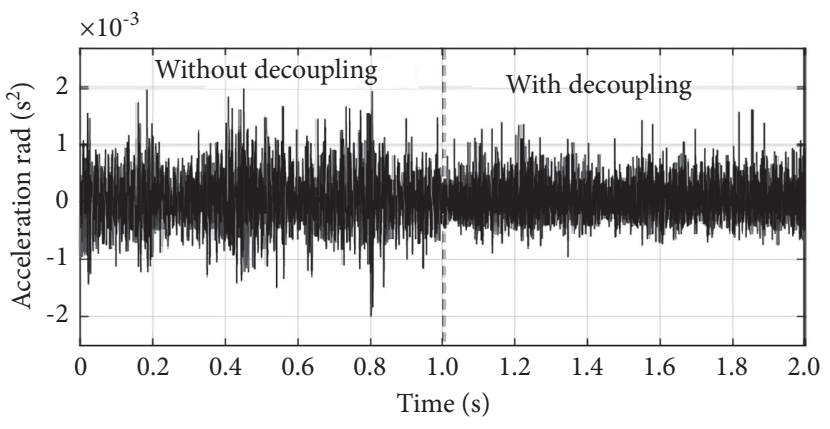

(a)

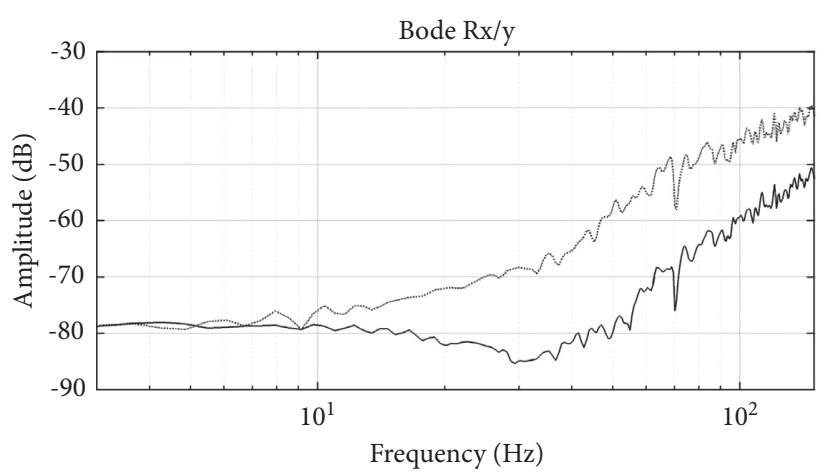

Without decoupling With decoupling

(b)

Figure 7: Comparison of random signal before and after decoupling without load. (a) Acceleration and (b) frequency domain waveform.

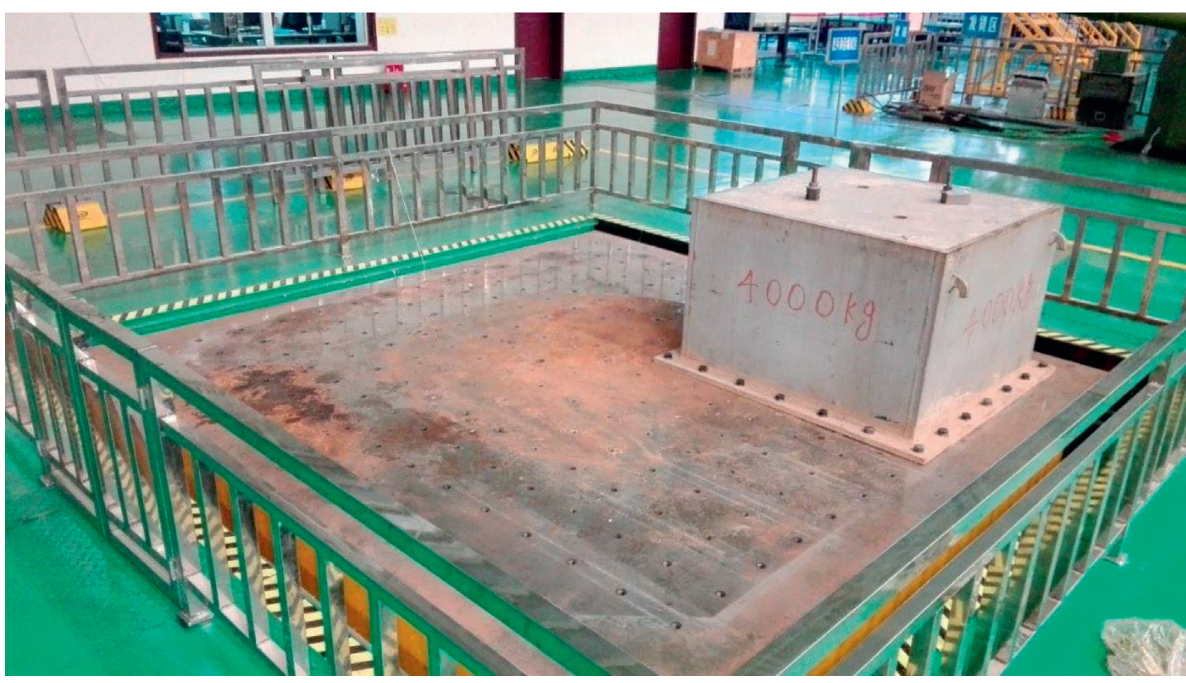

Figure 8: Multiaxis hydraulic servo shaking table with eccentric load.

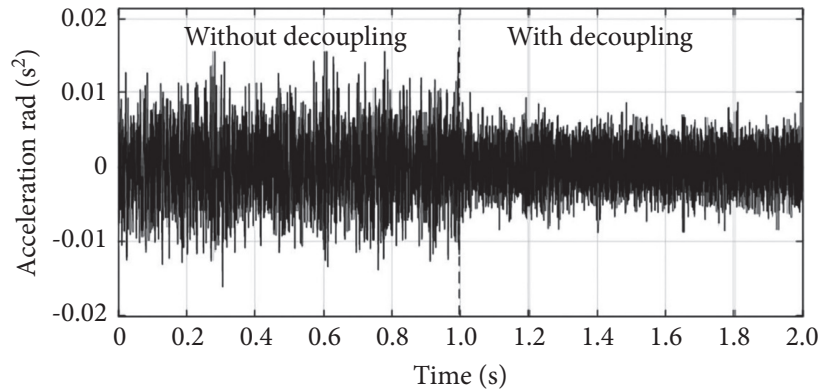

(a)

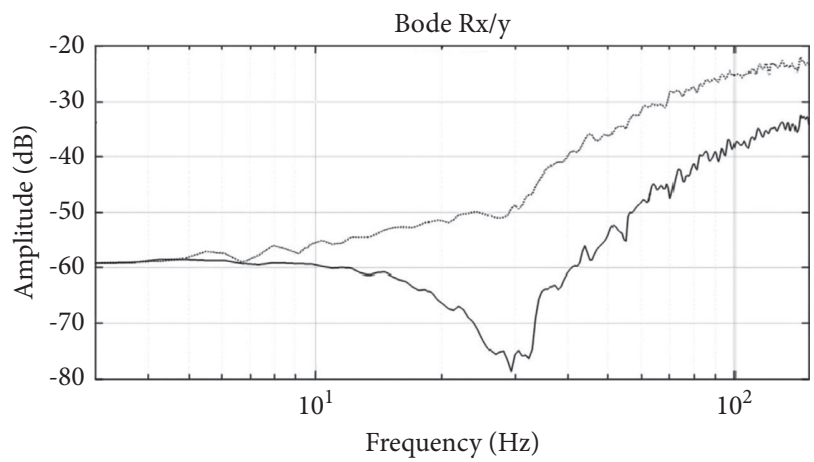

...... Without decoupling

— With decoupling

Figure 9: Comparison of random signal before and after decoupling with eccentric load. (a) Acceleration and (b) frequency domain waveform. 


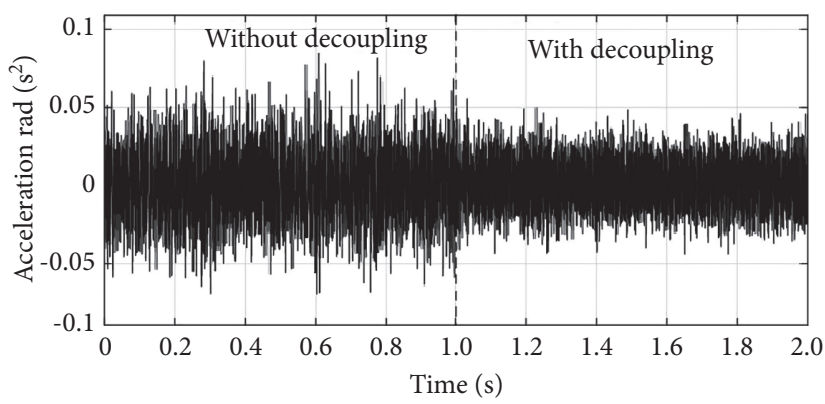

(a)

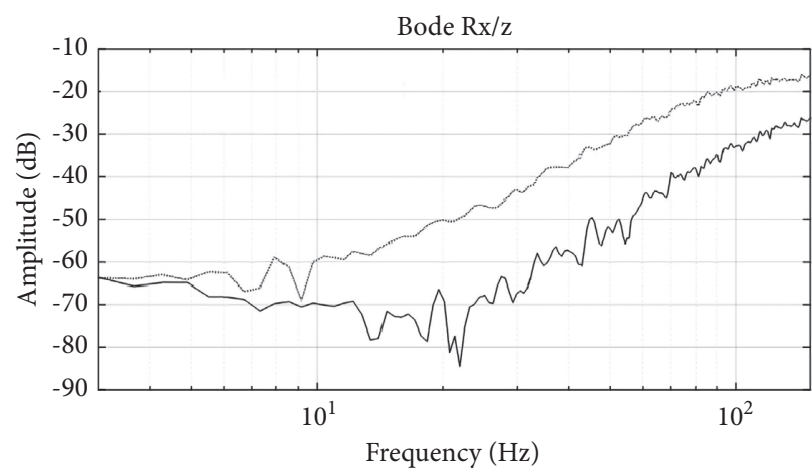

Without decoupling

With decoupling

(b)

FIGURE 10: Comparison of random signal before and after decoupling with eccentric load. (a) Acceleration and (b) frequency domain waveform.

signal is a random acceleration signal with a peak value of $2.2 \mathrm{~m} / \mathrm{s}^{2}$ in the direction of $Z$ degree of freedom. It can be seen from time domain that coupling signal output appeared in the direction of $R x$. Coupled output of the system will be reduced after decoupling controller is added. As can be seen from the frequency domain, the $Z$ degree of freedom direction output coupling with the input $\mathrm{Rx}$ direction is relatively large when the system has an eccentric load in the X/ $Y / Z$ direction, and the decoupling controller can reduce the coupling of $10 \mathrm{~dB}$ on average.

5.2. Experimental Results. The center of load is higher than the center of the upper surface of the platform $0.33 \mathrm{~m}$. The center of load of the platform is below the center of the surface of the platform $0.31 \mathrm{~m}$. The control point is the plane center formed by the upper hinge point of the horizontal hydraulic cylinder, which is $0.212 \mathrm{~m}$ from the upper surface of the platform. The eccentric value of eccentric load in $Z$ direction is $0.542 \mathrm{~m}$. The center of load below the control point is $0.98 \mathrm{~m}$.

The Bode diagram of $R x / y$ before and after decoupling with the random acceleration signal with a peak value of $0.4 \mathrm{~g}$ as input in the direction of $Y$ degrees of freedom is shown in Figure 11. It can be seen from Figure 10 that the peak value of the coupling before decoupling appears at about $50 \mathrm{~Hz}$ with a value of $-11.6 \mathrm{~dB}$, while the peak value after decoupling remains at about $50 \mathrm{~Hz}$ with a value of $-15.1 \mathrm{~dB}$. The coupling peak reduces by $3.5 \mathrm{~dB}$, but it can aggravate the coupling effect of some decoupling controllers beyond the peak value. The difference between the simulation result and the actual results lies in the fact that the center of mass of the platform in the simulation is considered as the control point of the shaking table, and the only reason for the coupling is the load of the platform. But for the actual system, the center of mass of the platform does not coincide with the control point rather than the below of control point. The calculation of the mass center and geometric relation of the use of numerical value is based on the design calculation, but the center of mass can be offset in the

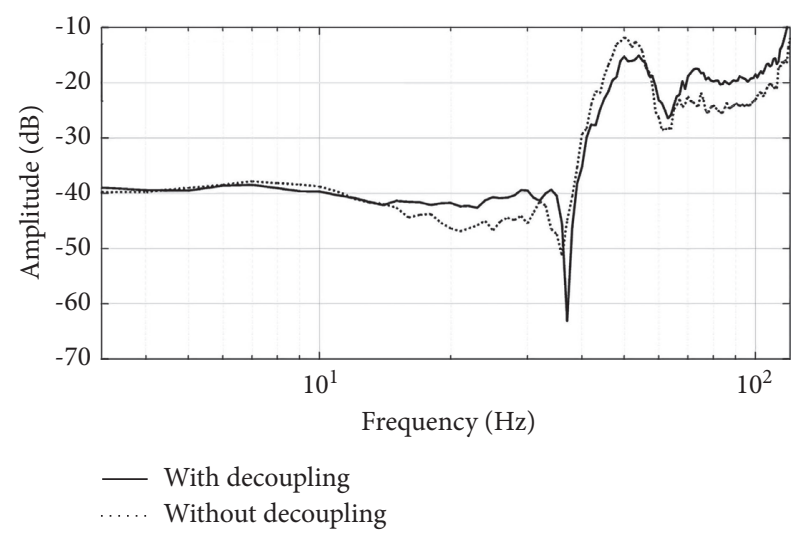

FIgURE 11: Comparison of random signal before and after decoupling with eccentric load. (a) Acceleration and (b) frequency domain waveform.

actual construction process. If the center of mass is shifted downward, then the system platform load and the combined center of mass of the platform are near the control point, so the coupling caused by the dynamics itself is relatively small, and the decoupling controller aggravates the coupling output of the system.

The center of load is higher than the surface of the platform $0.33 \mathrm{~m}$, and the offset of $X$ direction and $Y$ direction is $0.86 \mathrm{~m}$, respectively. The center of load is below the center of the platform of the surface that is $0.31 \mathrm{~m}$. The eccentric load in the coordinates of control points for the origin of conjoined coordinate system is $0.86 \mathrm{~m}, 0.86 \mathrm{~m}$, and $0.542 \mathrm{~m}$, and the center of load is $0.98 \mathrm{~mm}$ below the control point.

In this case, the maximum coupling direction will appear in the direction of $R x / z$ and $R y / z$. That is, the system will have coupling acceleration signal output in the direction of $R x$ and $R y$ when the system has acceleration signal input in the direction of $Z$ degrees of freedom. The above coupled mass matrix is brought into the controller based on dynamic decoupling designed in Section 4. The random acceleration signal with a peak value of $0.4 \mathrm{~g}$ is input in the direction of $Z$ 


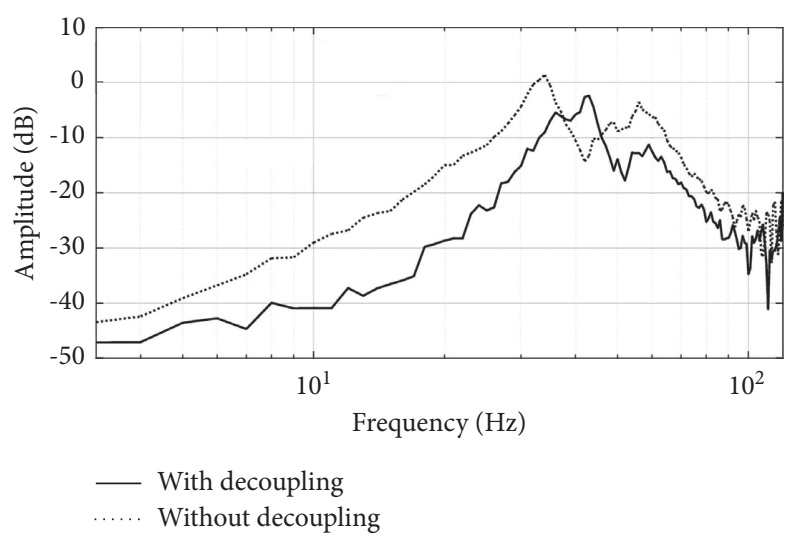

Figure 12: Comparison of random signal before and after decoupling with eccentric load.

degrees of freedom, and then the Bode diagram of $R x / Z$ and $R y / Z$ before and after decoupling is obtained as shown in Figures 12 and 13.

As can be seen from Figures 12 and 13, the peak value of the coupling before decoupling appears between $30 \mathrm{~Hz}$ and $40 \mathrm{~Hz}$, and the maximum peak value moves to the right after decoupling, reducing the coupling by $6 \mathrm{~dB}$ on average. The corresponding amplitude after decoupling in the time domain is $50.12 \%$ of that before decoupling. It can be seen from Figure 13 that the peak value of the coupling before decoupling is between $45 \mathrm{~Hz}$ and $60 \mathrm{~Hz}$. After decoupling, the average coupling is less than $8 \mathrm{~dB}$, and the corresponding amplitude after decoupling in the time domain is about $39.81 \%$. It can be seen that, before decoupling, the output coupling in these two coupling degrees of freedom directions is relatively large, and the effect is also obvious.

The comparison of decoupling effect is listed in Table 2. From the geometric relationship, it can be seen that the coupling size should be the same in $R x$ and $R y$ direction when random signals with the same peak value are input in $z$ direction. However, due to the different parameters of the control system in each direction of degrees of freedom and the difference of actuators, the coupling characteristics of these two coupled degrees of freedom are not exactly the same.

With the comparison of the latest related work, Dai et al. [28] proposed a decoupling control method in modal space for six-degree-of-freedom parallel mechanisms. In their research, the coupling motion could reduce by $9.42 \%$ IN RX and $19.22 \%$ in RY under sinusoid input signal with $2 \mathrm{~Hz}$, $0.02 \mathrm{~m}$ (amplitude in $X$ direction) and $3 \mathrm{~Hz}, 0.03 \mathrm{~m}$ (amplitude in $Y$ direction), respectively. It should be noted that the model in this article is a little bit different from ours; even they are both parallel mechanisms, which would have significant coupling phenomenon during either operation. The different structure and design parameters would cause diverse performance in coupling and decoupling effects. But both confirm the necessity and effectiveness of decoupling control.

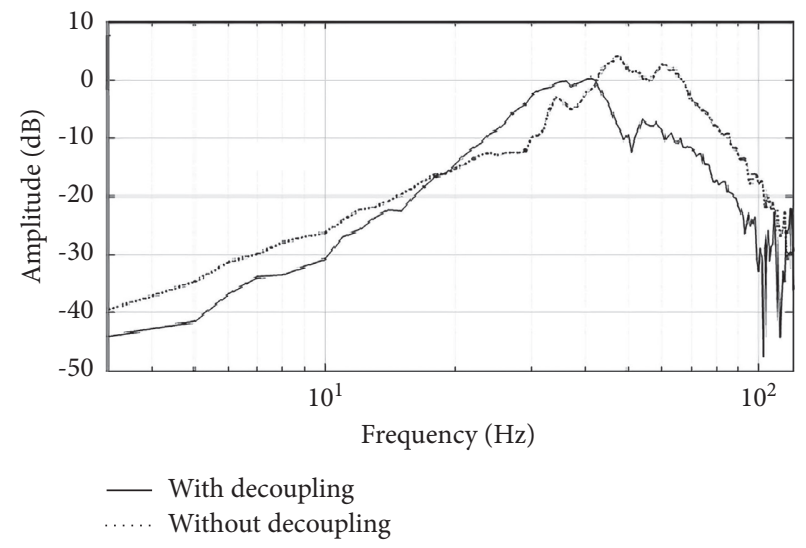

FIgURE 13: Comparison of random signal before and after decoupling with eccentric load.

TABle 2: Comparison of decoupling effect.

\begin{tabular}{lcc}
\hline Degree & Decoupling effect $(\mathrm{dB})$ & Decoupling effect (\%) \\
\hline$R x / z$ & $-6 \mathrm{~dB}$ & $-49.88 \%$ \\
$R y / z$ & $-8 \mathrm{~dB}$ & $-60.19 \%$ \\
\hline
\end{tabular}

\section{Conclusions}

In this paper, a decoupling controller based on dynamic model is introduced to realize decoupling for the independent control of the multiaxis hydraulic servo shaking table. On the basis of analysis of working principles, the kinematics mode and dynamic model of the multiaxis hydraulic servo shaking table are all established. Basic causes of coupling phenomenon of shaking table are summarized. The suggestions are given to address each reason. In particular, the decoupling control strategy based on dynamic model is proposed to compensate the system coupling caused by dynamic characteristics. As the multiaxis hydraulic servo shaking table is redundant, the modal space of the multiaxis hydraulic servo shaking table is established to transform the coupled system into eight independent systems to realize the independent control of eight actuators. Simulation and experimental results show that the proposed control strategy can improve the control accuracy and decoupling characteristic effective. With eccentric load, the coupling could reduce $-49.88 \%(R x / z)$ and $-60.19 \%(R y /$ $z$ ) after decoupling control.

\section{Future Work}

The research work in future steps would include inputting composite singles of different degrees of freedom. The composite signal is a more effective approach to represent objective single with higher precision. However, the combination of different degrees of freedom singles may bring a more complex coupling. The decoupling control of composite singles to achieve the higher precision deserves to be studied. 


\section{Data Availability}

All the data used to support the findings of this study are included within the relevant figures and tables in the article.

\section{Conflicts of Interest}

The authors declare that there are no conflicts of interest regarding the publication of this study.

\section{Acknowledgments}

This research was supported by the National Natural Science Foundation of China (Grant no. 51105094), National Key R\&D Program of China (Grant no. 2020YFB2009701), and the Science Foundation for Post Doctorate research Foundation of Heilongjiang Province.

\section{References}

[1] H. E. Blomgren, P. Shiling, J. Zhibin, and P. Josh, "Full-scale shake table testing of cross-laminated timber rocking shear walls with replaceable components," Journal of Structural Engineering, vol. 145, no. 10, pp. 1-14, 2019.

[2] J. Liu, B. Qiao, X. Zhang, R. Yan, and X. Chen, “Adaptive vibration control on electrohydraulic shaking table system with an expanded frequency range: theory analysis and experimental study," Mechanical Systems and Signal Processing, vol. 132, pp. 122-137, 2019.

[3] N. Nakata, "A multi-purpose earthquake simulator and a flexible development platform for actuator controller design," Journal of Vibration and Control, vol. 18, no. 10, pp. 15521560, 2012.

[4] J. J. Yao, Z. S. Wan, and Y. Fu, “Acceleration harmonic estimation in a hydraulic shaking table using water cycle algorithm," Shock and Vibration, vol. 2018, Article ID 7278589, 12 pages, 2018.

[5] J. J. Yao Jianjun, S. Hu, W. Fu, and J. Han, "Harmonic cancellation for electro-hydraulic servo shaking table based on LMS adaptive algorithm," Journal of Vibration and Control, vol. 17, no. 12, pp. 1862-1868, 2011.

[6] Z. Chen, C. Li, B. Yao, M. Yuan, and C. Yang, "Integrated coordinated/synchronized contouring control of a dual-linear-motor-driven gantry," IEEE Transactions on Industrial Electronics, vol. 67, no. 5, pp. 3944-3954, 2020.

[7] Y. Tang, Z. Zhu, and G. Shen, "Design and experimental evaluation of feedforward controller integrating filtered-x LMS algorithm with applications to electro-hydraulic force control systems," Proceedings of the Institution of Mechanical Engineers - Part C: Journal of Mechanical Engineering Science, vol. 230, no. 12, pp. 1951-1967, 2016.

[8] G. Shen, X. Li, Z. Zhu, Y. Tang, W. Zhu, and S. Liu, "Acceleration tracking control combining adaptive control and off-line compensators for six-degree-of-freedom electro-hydraulic shaking tables," ISA Transactions, vol. 70, pp. 322-337, 2017.

[9] S. Kockanat, "Acceleration harmonic estimation using an approach based artificial bee colony algorithm: a hydraulic shaking table Application," in Proceedings of the 2019 27th Signal Processing and Communications Applications Conference.

[10] S. H. Larbi, B. Nouredine, B. Hacine, C. Khireddine, and B. Mohammed, "Earthquake ground motion matching on a small electric shaking table using a combined NN-pdff controller," Shock and Vibration, vol. 2020, Article ID 7260590, 14 pages, 2020.

[11] Y. Liu and W. Tao, G. Guofang and G. Rujun, Present status and prospect of high-frequency electro-hydraulic vibration control technology," Chinese Journal of Mechanical Engineering, vol. 32, no. 1, 2019.

[12] G. Shen, G. Li, W. Zang, X. Li, and Y. Tang, "Modal space feedforward control for electro-hydraulic parallel mechanism,” Ieee Access, vol. 7, pp. 39751-39761, 2019.

[13] Y. Tagawa and K. Kajiwara, "Controller development for the E-Defense shaking table," Proceedings of the Institution of Mechanical Engineers - Part I: Journal of Systems \& Control Engineering, vol. 221, no. I2, pp. 171-181, 2007.

[14] G. F. Guan and A. R. Plummer, "Acceleration decoupling control of 6 degrees of freedom electro-hydraulic shaking table," Journal of Vibration and Control, vol. 25, no. 21-22, pp. 2758-2768, 2019.

[15] A. R. Plummer, "Model-based motion control for multi-axis servohydraulic shaking tables," Control Engineering Practice, vol. 53, pp. 109-122, 2016.

[16] G. Shen, Z. Zhencai, and L. Xiang, "Experimental evaluation of acceleration waveform replication on electrohydraulic shaking tables: a review," International Journal of Advanced Robotic Systems, vol. 13, 2016.

[17] C. Yang, Z. Qu, and J. Han, "Decoupled-space control and experimental evaluation of spatial electrohydraulic robotic manipulators using singular value decomposition algorithms," IEEE Transactions on Industrial Electronics, vol. 61, no. 7, pp. 3427-3438, 2014.

[18] J. Zhao, Z. Wang, C. Zhang, C. Yang, W. Bai, and Z. Zhao, "Modal space three-state feedback control for electro-hydraulic servo plane redundant driving mechanism with eccentric load decoupling," ISA Transactions, vol. 77, pp. 201-221, 2018.

[19] W. Deng and J. Yao, "Adaptive integral robust control and application to electromechanical servo systems," ISA Transactions, vol. 67, pp. 256-265, 2017.

[20] X. Wang, D. Cong, Z. Yang, S. Xu, and J. Han, "Iterative learning control with complex conjugate gradient optimization algorithm for multiaxial road durability test rig," Proceedings of the Institution of Mechanical Engineers - Part C: Journal of Mechanical Engineering Science, vol. 233, no. 7, pp. 2349-2360, 2019.

[21] G. Shen, Z. Zhu, Y. Tang et al., "Combined control strategy using internal model control and adaptive inverse control for electro-hydraulic shaking table," Proceedings of the Institution of Mechanical Engineers - Part C: Journal of Mechanical Engineering Science, vol. 227, no. 10, pp. 2348-2360, 2013.

[22] L. P. Zhang, C. Dacheng, Y. Zhidong, Z. Yanyan, and H. Junwei, "Optimal design and hybrid control for the electrohydraulic dual-shaking table system," Applied Sciences-Basel, vol. 6, no. 220, pp. 1-32, 2016.

[23] T. Tian, H. Jiang, Z. Tong, J. He, and Q. Huang, “An inertial parameter identification method of eliminating system damping effect for a six-degree-of-freedom parallel manipulator," Chinese Journal of Aeronautics, vol. 28, no. 2, pp. 582-592, 2015.

[24] G. F. Guan, "Servo control based on kinematics analysis of 6 DOF hydraulic shaker," in Proceedings of the Seventh International Conference on Fluid Power Transmission and Control, Y. X. Lu, Q. F. Wang, and B. F. Ju, Eds., pp. 413-416, World Publishing Corporation, Hangzhou, China, 2009. 
[25] J. J. Yao, W. Zhenshuai, Z. Yue, Y. Jie, Q. Chen, and F. Yu, "Resonance suppression for hydraulic servo shaking table based on adaptive notch filter," Shock and Vibration, vol. 2019, Article ID 9407520, 12 pages, 2019.

[26] J. J. Yao, Z. S. Wan, and Y. Fu, "Acceleration harmonic estimation for hydraulic servo shaking table by using simulated annealing algorithm," Applied Sciences-Basel, vol. 8, no. 4, 2018.

[27] J. Zhao, G. Shen, C. Yang, W. Zhu, and J. Yao, "A robust force feed-forward observer for an electro-hydraulic control loading system in flight simulators," ISA Transactions, vol. 89, pp. 198-217, 2019.

[28] X. Dai, S. Song, and D. Gong, "Modal space decoupled control of six-degree-of-freedom parallel mechanisms," Journal of Harbin Engineering University, vol. 41, no. 8, pp. 1237-1243, 2020 . 